\title{
Heavy-atom antiferromagnet GdBiTe: an interplay of magnetism and topology in a symmetry-protected topological semimetal
}

\section{Supporting Information}

Paul Gebauer, ${ }^{1,2}$ Hagen Poddig, ${ }^{1}$ Laura T. Corredor-Bohorquez, ${ }^{2}$ Tatiana V.

Menshchikova, ${ }^{3}$ Igor P. Rusinov, ${ }^{3}$ Pavlo Golub, ${ }^{4}$ Federico Caglieris, ${ }^{5}$ Christopher Benndorf, 6 Tobias Lindemann, ${ }^{6}$ Evgueni V. Chulkov, ${ }^{7,8}$ Anja U. B. Wolter, ${ }^{2,10}$ Bernd Büchner, ${ }^{2,9,10}$ Thomas Doert, ${ }^{1}$ and Anna Isaeva ${ }^{2,9,10^{*}}$

\footnotetext{
${ }^{1}$ Faculty of Chemistry and Food Chemistry, Technische Universität Dresden, 01062 Dresden, Germany, ${ }^{2}$ Institute for Solid State Research, Leibniz IFW Dresden, 01069 Dresden, Germany, ${ }^{3}$ Tomsk State University, 634050 Tomsk, Russia, ${ }^{4}$ Department of Theoretical Chemistry, J. Heyrovsky Institute of Physical Chemistry, 18223 Prague, Czech Republic, ${ }^{5}$ CNR-SPIN, 16152, Genova, Italy, ${ }^{6}$ Institute for Mineralogy, Crystallography and Materials Science, Faculty of Chemistry and Mineralogy, Leipzig University, 04275 Leipzig, Germany, ${ }^{7}$ National Research University Higher School of Economics, 101000 Moscow, Russia, ${ }^{8}$ Donostia International Physics Center (DIPC), 20018 SanSebastian/Donostia, Spain, ${ }^{9}$ Institute of Solid State and Materials Physics, Technische Universität Dresden, 01062 Dresden, Germany, ${ }^{10}$ Würzburg-Dresden Cluster of Excellence ct.qmat, 97074 Würzburg and 01062 Dresden, Germany
} 


\section{Summary of the attempts to synthesize GdBiTe 3}

To obtain $\mathrm{GdBiTe}_{3}$ we initially followed the strategies reported in [15-17]. Solid-state reactions between $\mathrm{Gd}_{2} \mathrm{Te}_{3}$ and $\mathrm{Bi}_{2} \mathrm{Te}_{3}$ taken in a molar $1: 1$ ratio and annealed at various temperatures within the $600-850{ }^{\circ} \mathrm{C}$ range yielded

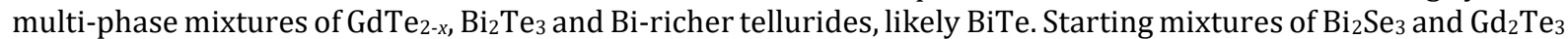
annealed at $950{ }^{\circ} \mathrm{C}$ provided a similar outcome. Accordingly, DSC studies of the stoichiometric $\mathrm{Gd}_{2} \mathrm{Te}_{3}$ and $\mathrm{Bi}_{2} \mathrm{Te}_{3}$ mixtures cycled up to 800 or $950^{\circ} \mathrm{C}$ and back down to room temperature showed only the melting signal of bismuth telluride(s) at $582{ }^{\circ} \mathrm{C}$ (heating curve) and $584{ }^{\circ} \mathrm{C}$ (cooling curve). Besides, a small portion of Gd $2 \mathrm{Te}_{3}$ likely reacted with the quartz walls producing $\mathrm{Gd}_{2} \mathrm{TeO}_{2}$ as detected in the powder X-ray diffraction (PXRD) patterns. In addition, growth from KI flux was attempted by slow cooling at the rate of $2 \mathrm{~K} / \mathrm{min}$ from $850{ }^{\circ} \mathrm{C}$ down to $375^{\circ} \mathrm{C}$, resulting in $\mathrm{Bi}_{2} \mathrm{Te}_{3}$ and $\mathrm{BiTe}$ in form of small crystals and melting beads. The Gd was bound in more or less amorphous powder. No change in the phase composition was observed after a long-term annealing of the starting $\mathrm{GdTe}_{2-x}$ and $\mathrm{Bi}_{2} \mathrm{Te}_{3}$ (2:1 ratio) mixture.

Beside we tried multiple other synthetic routes starting from various reactants. Solid-state reactions of the stoichiometric mixtures of the elements led to the formation of $\mathrm{Bi}_{2} \mathrm{Te}_{3}$ and $\mathrm{GdTe}_{3}$ at $270{ }^{\circ} \mathrm{C}$, and of $\mathrm{BiTe}$ and $\mathrm{GdTe}_{2}-$ $x$ at $470{ }^{\circ} \mathrm{C}$. A mixture of $\mathrm{Bi}_{2} \mathrm{Te}_{3}$ and $\mathrm{GdTe}_{3}$ taken in $1: 1$ molar ratio was subjected to long-term annealing at $850{ }^{\circ} \mathrm{C}$ or $950^{\circ} \mathrm{C}$ and was explored by thermochemical analysis. No reaction was observed, the PXRD after the treatment showed only the starting materials. Likewise, we observed no chemical reaction between $\mathrm{GdTe}_{2}$ and $\mathrm{Bi}_{2} \mathrm{Te}_{3}$ taken in a 1:1 molar ratio. When $\mathrm{GdTe} 2$ reacts with $\mathrm{Bi}$ at $950{ }^{\circ} \mathrm{C}$ for several days, a phase mixture of $\mathrm{Bi}_{2} \mathrm{Te}_{3}$ (and/or $\mathrm{Bi}$ richer telluride), $\mathrm{Gd}_{2} \mathrm{Te}_{3}$ and bismuth is obtained. The respective DSC study shows the melting signals of $\mathrm{Bi}_{2} \mathrm{Te}_{3}$ at ca. $580^{\circ} \mathrm{C}$. Reacting $\mathrm{GdTe}_{3}$ with Bi results in a mixture of $\mathrm{GdTe}_{2}$ and BiTe.

Chemical transport reactions with iodine in various temperature gradients were performed. Unfortunately, transport of bismuth and gadolinium tellurides enhances in very different temperature ranges, e.g. while $\mathrm{Bi}_{2} \mathrm{Te}_{3}$ migrate most efficiently from $500{ }^{\circ} \mathrm{C}$ to $400{ }^{\circ} \mathrm{C}$, GdTe 2 migrates from $900{ }^{\circ} \mathrm{C}$ to $800{ }^{\circ} \mathrm{C}$ Setting the temperature gradient somewhat between 500 and $800{ }^{\circ} \mathrm{C}$ we were able to grow BiTeI crystals only. Syntheses with $\mathrm{GdCl}_{3}$ and $\mathrm{Bi}_{2} \mathrm{Te}_{3}$ mixtures with various annealing regimes did not result in any changes of the initial phase composition. Temperature gradients chosen between 450 and $600{ }^{\circ} \mathrm{C}$ favored the crystal growth of $\mathrm{Bi}_{2} \mathrm{Te}_{3}$.

\section{Powder pattern}

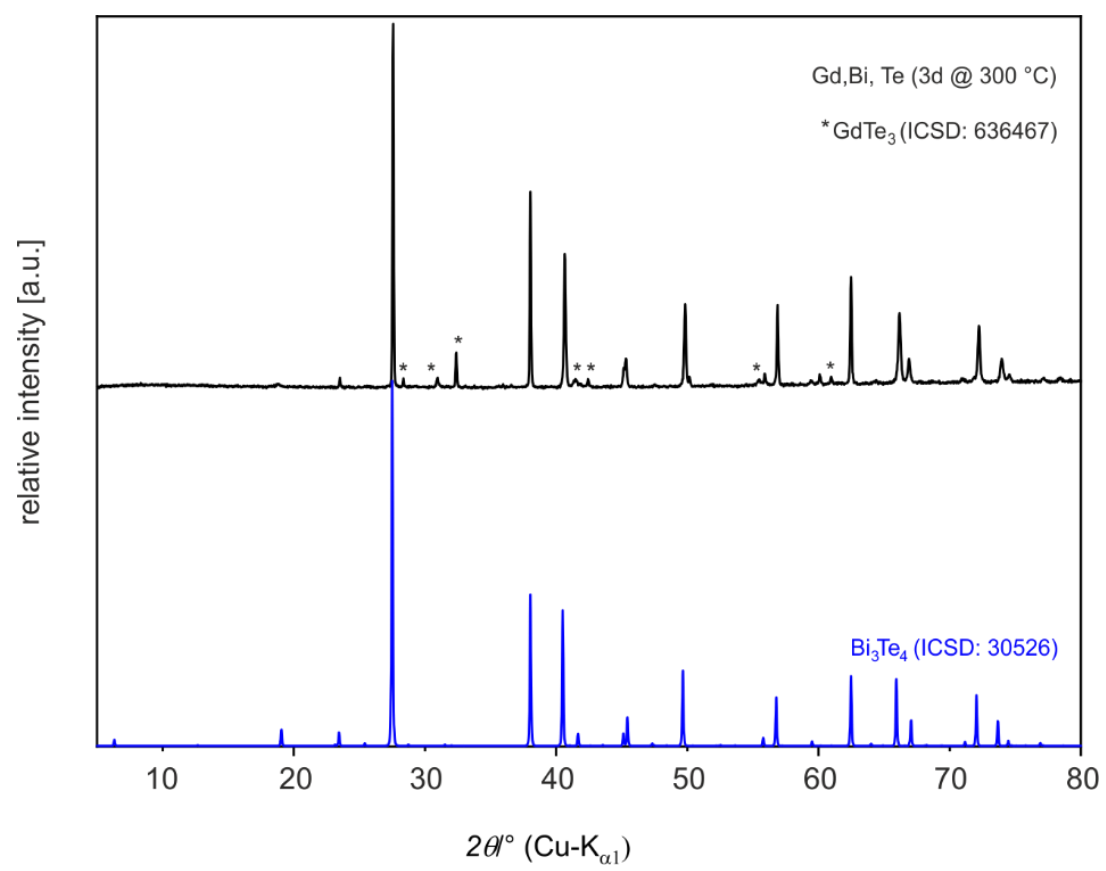

Figure S1. PXRD pattern of an equimolar mixture of $\mathrm{Gd}, \mathrm{Bi}$, Te annealed at $300{ }^{\circ} \mathrm{C}$ for 3 days. At this low temperature no ternary phase is formed. 


\section{Crystal Structure Elucidation of GdBiTe and LaBiTe}

Table S1. Coefficients $U_{\mathrm{ij}}\left(\mathrm{in} \AA^{2}\right.$ ) of the tensors of the anisotropic displacement and equivalent isotropic displacement parameters for the atoms in GdBiTe. For all atoms $U_{23}=U_{13}=0 . U_{\text {eq }}$ is defined as one third of the trace of the orthogonalized $U_{\mathrm{ij}}$ tensor.

\begin{tabular}{llllll}
\hline Atom & $U_{11}$ & $U_{22}$ & $U_{33}$ & $U_{12}$ & $U_{\text {eq }}$ \\
Gd1 & $0.00305(6)$ & $0.00305(6)$ & $0.0036(1)$ & 0 & $0.00324(5)$ \\
Bi1 & $0.00370(5)$ & $0.00370(5)$ & $0.00471(8)$ & 0 & $0.00404(5)$ \\
Te1 & $0.00285(7)$ & $0.00285(7)$ & $0.0039(1)$ & 0 & $0.00320(6)$ \\
\hline
\end{tabular}

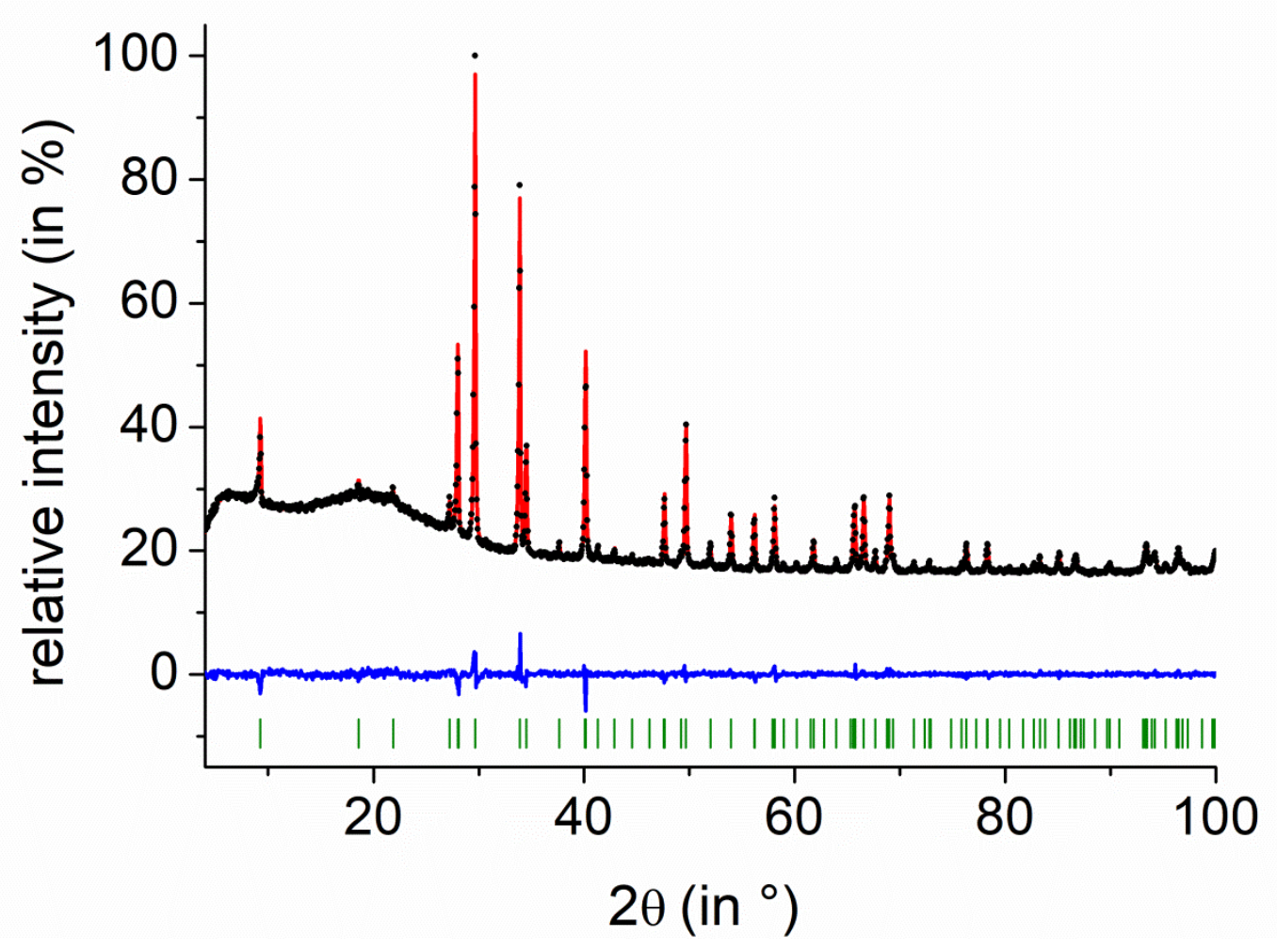

Figure S2. Rietveld refinement of LaBiTe (Huber G670 diffractometer, $\lambda=154.06 \mathrm{pm} ; R_{\mathrm{wp}}=1.63 \%$, GooF $=0.65$, space group $P 4 / \mathrm{nmm}, R_{\mathrm{Bragg}}=1.70 \%, 31$ parameters (thereof 18 background), $a=4.48814(9) \AA, c=9.5501(3) \AA$, $V=192.371 \AA^{3}, \rho=8.209 \mathrm{~g} \mathrm{~cm}^{-3}$ ). The experimental data is depicted by black dots, the calculated diffraction pattern in red. The difference plot is drawn in blue. Vertical green lines show the position of the Bragg reflections. 


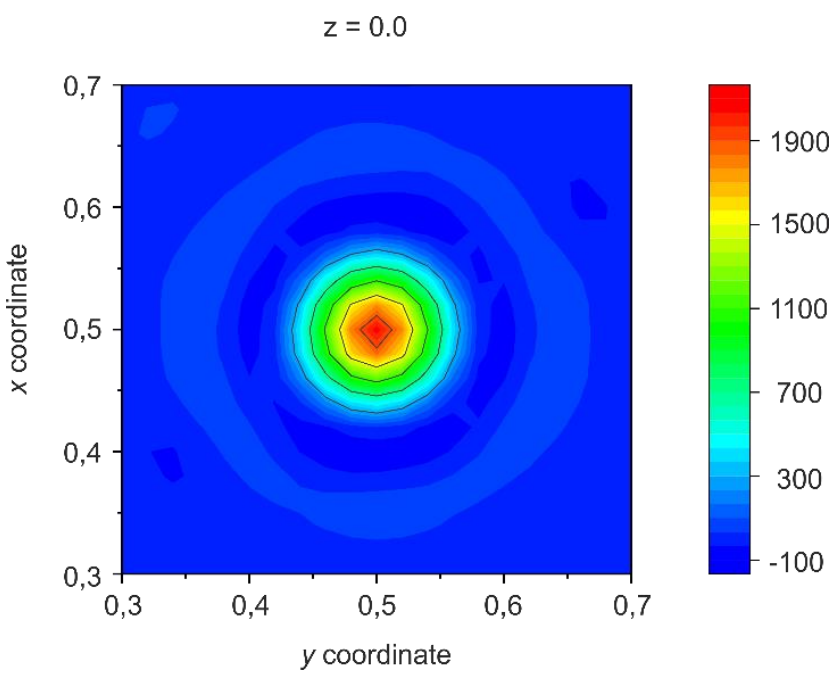

Figure S3. Fourier section around Bi1 in GdBiTe. The electron density is drawn in contour line steps of $400 \mathrm{eA}^{-3}$.

\section{Wilson loop spectra and surface electronic structures of GdBiTe when $\overrightarrow{\mathcal{M}} \| z$}

\section{AFM-I, $\overrightarrow{\mathcal{M}} \| z$}

(a)

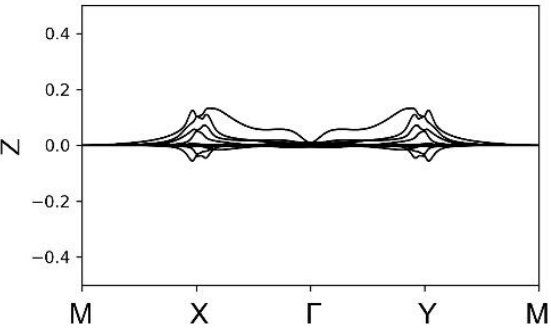

(c)

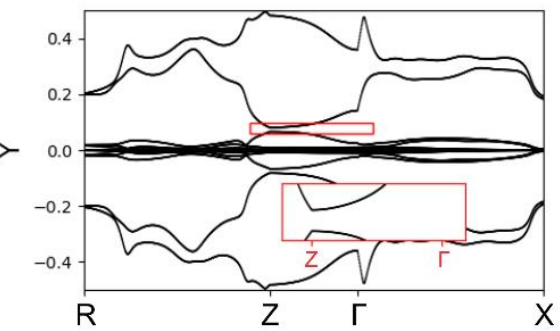

(e)

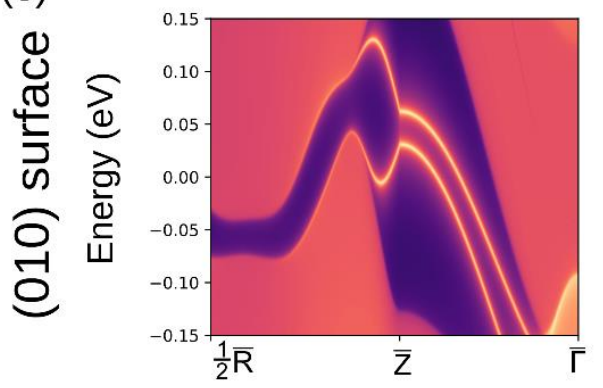

(b)

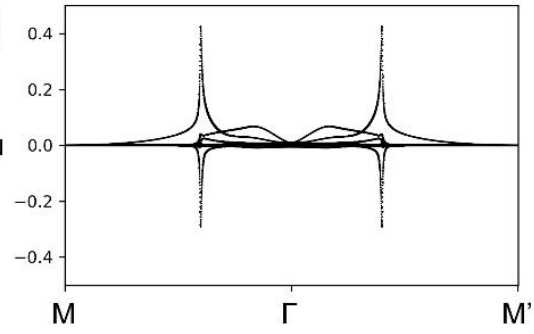

(d)

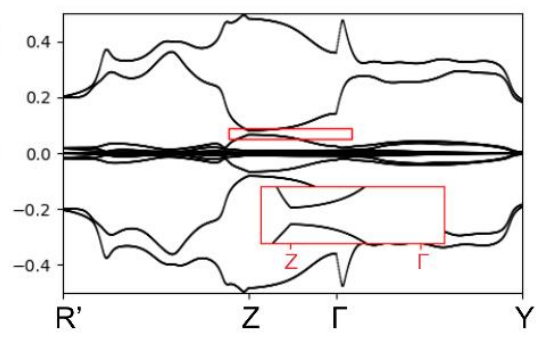

(f)

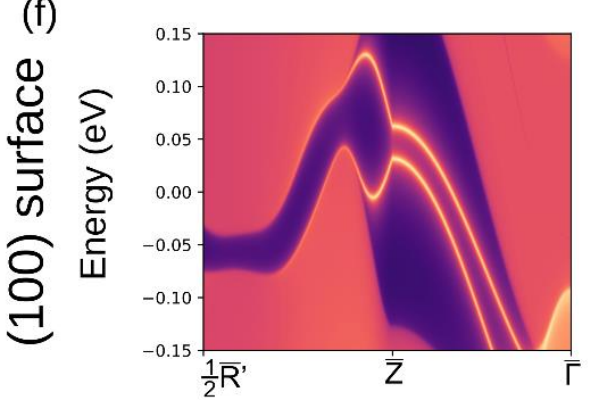

Figure S4. (a-d) Wilson loop spectra and (e, f) surface electronic spectra of the AFM-I antiferromagnetic phase with magnetic moments oriented out-of-plane. 


\section{Evolution of the GdBiTe bulk electronic structure as a function of the Hubbard parameter $U$}

Magnetic properties of GdBiTe arise from the Gd f-electrons. According to our theoretical calculations, these electrons form two groups of almost flat bands in the valence and in the conduction parts of the band structure. These groups are localized at around -5.5 and $0.5 \mathrm{eV}$, respectively, at $\mathrm{U}=0 \mathrm{eV}$ (Fig. S5). Close to the Fermi level, other electronic states do not hybridize with the f-states. An increase of the Hubbard parameter $U$ leads to a downward (upward) shift in the energy of the valence (conduction) f-bands, respectively, without any changes in the dispersion of the other bands. As a result, the identified (topological) features and the conclusions made in the manuscript do not alter with the Hubbard parameter.
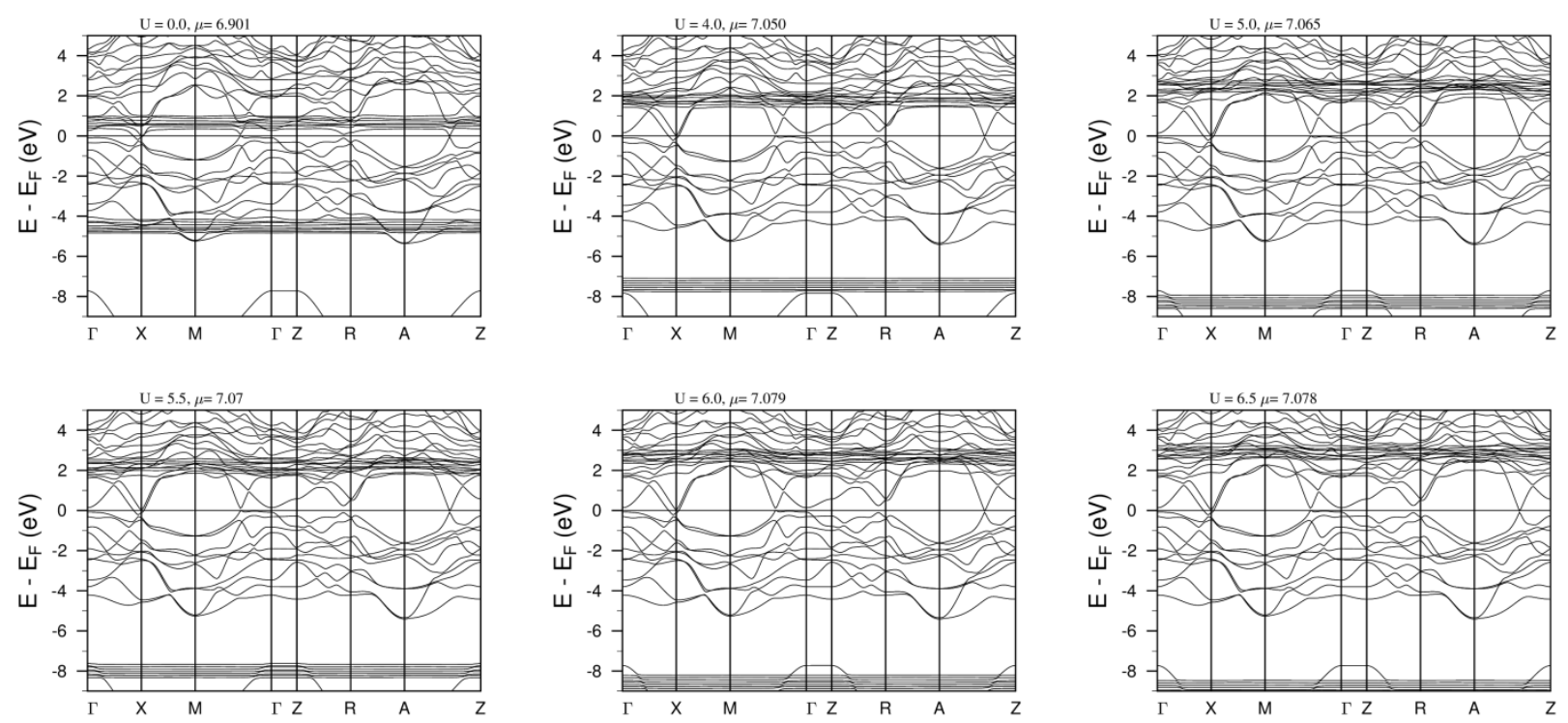

Figure S5. Bulk electronic structure of GdBiTe at various $U$. $U$ - the Hubbard parameter, $\mu$ - the calculated magnetic moment $\left(\mu_{B}\right)$ per Gd atom. 